\title{
Molecular and immunologic markers of kidney cancer-potential applications in predictive, preventive and personalized medicine
}

\author{
Amanda Mickley ${ }^{1}$, Olga Kovaleva², Julia Kzhyshkowska ${ }^{1,3,4}$ and Alexei Gratchev 2,4,5*
}

\begin{abstract}
Kidney cancer is one of the deadliest malignancies due to frequent late diagnosis (33\% or renal cell carcinoma are metastatic at diagnosis) and poor treatment options. There are two major subtypes of kidney cancer: renal cell carcinoma (RCC) and renal pelvis carcinoma. The risk factors for RCC, accounting for more than $90 \%$ of all kidney cancers, are smoking, obesity, hypertension, misuse of pain medication, and some genetic diseases. The most common molecular markers of kidney cancer include mutations and epigenetic inactivation of von Hippel-Lindau (VHL) gene, genes of vascular endothelial growth factor (VEGF) pathway, and carbonic anhydrase IX (CIAX). The role of epigenetic pathways, including DNA methylation and chromatin structure remodeling, was also demonstrated. Immunologic properties of RCC enable this type of tumor to escape immune response effectively. An important role in this process is played by tumor-associated macrophages that demonstrate mixed M1/M2 phenotype. In this review, we discuss molecular and cellular aspects for RCC development and current state of knowledge allowing personalized approaches for diagnostics and prognostic prediction of this disease. A set of macrophage markers is suggested for the analysis of the association of macrophage phenotype and disease prognosis.
\end{abstract}

Keywords: Kidney, Renal cell carcinoma, Mutation, Epigenetics, Tumor-associated macrophage, Predictive, preventive and personalized medicine, Biomarker panel, Metastatic disease, Molecular target, Immune response, Disease prognosis

\section{Introduction}

Kidney cancer is one of the most common and deadly malignancies, with approximately 58,000 new cases and 13,000 deaths estimated to have occurred in the USA in 2010 (http://www.cancer.gov/cancertopics/types/kidney). There are two main types of kidney cancer, which includes renal cell carcinoma (RCC) and renal pelvis carcinoma. RCC is the most prevalent, accounting for $90 \%$ of all kidney cancers with $33 \%$ of these cases being metastatic at diagnosis [1]. Risk factors for the development of RCC include cigarette smoking, obesity, continued misuse of pain medications, acquired cystic kidney disease, hypertension, and other genetic diseases [2, 3].

\footnotetext{
* Correspondence: alexei.gratchev@gmail.com

${ }^{2}$ Blokhin Cancer Research Center, Moscow, Russia

${ }^{4}$ Laboratory for Translational Cellular and Molecular Biomedicine, Tomsk State University, Tomsk, Russia

Full list of author information is available at the end of the article
}

The major problems in treating RCC are late diagnosis and poor response to available therapies.

RCC arises from the proximal renal tubular epithelium and involves several different cell types, such as clear cells, granular cells, and spindle cells. The proximal renal tubule plays an important role in homeostasis, regulating $\mathrm{pH}$, salt concentration, glucose concentration, and other substances by reabsorption into the blood or excretion with the urine [4]. There are both genetic and epigenetic alterations that are connected to RCC development. Genetic disorders leading to RCC and their genes of mutation can be seen in Table 1 [5]. The most prevalent genetic cause of RCC is von Hippel-Lindau syndrome, a disorder characterized by the development of vascular tumors such as RCC, hemangioblastomas of the central nervous system and pheochromocytoma [5, 6]. The von Hippel-Lindau (VHL) gene on chromosome 3 (3p25-26) has been identified as a tumor suppressor gene for this syndrome [5]. The VHL gene is inactivated in $75 \%$ of 
Table 1 Hereditary renal cell syndromes with genes of mutation

\begin{tabular}{ll}
\hline Syndrome & Gene (chromosome) \\
\hline von Hippel-Lindau & VHL (3p25-26) \\
Hereditary papillary renal carcinoma & C-Met proto-oncogene (7q31-34) \\
Hereditary leiomyomatosis RCC & Fumarate hydratase (1q42-43) \\
Birt-Hogg-Dubé & BHD1 (17p11) \\
Tuberous sclerosis & TSC1 (9q34) or TSC2 (16p13) \\
\hline
\end{tabular}

Modified from [5]

RCC cases [7]. Patients inherit one mutated allele and acquire a mutation in the second allele of the affected organs. Patients who have non-inherited Hippel-Lindau syndrome have acquired a mutation in both alleles [5].

\section{Molecular predictive and prognostic markers}

The three main predictive and prognostic markers used for RCC are VHL, vascular endothelial growth factor (VEGF), and carbonic anhydrase IX (CAIX) [3, 8]. The first of which, VHL, has been studied immensely as a biomarker. In a study by Yao et al., it was shown that the presence of VHL alterations is associated with better outcomes for patients with stages I-III clear cell RCC treated by nephrectomy [9]. Mutational analyses showed that almost $100 \%$ of related RCC patients have germ line VHL alterations; $42-57 \%$ of sporadic clear cell RCC patients have somatic intragenic mutations, and in 5-19\% of these tumors, aberrant hypermethylation of VHL has also been found [9]. In another study by Choueiri et al., it was found that patients with an alteration of the VHL gene had a better response to anti-VEGF therapy as opposed to those with the wild-type gene [10].

VEGF is an important ligand involved in tumor angiogenesis. When patients are effectively treated with VEGFtargeted therapy, VEGF levels in the serum rise and soluble VEGFR levels decrease. Therefore, these factors are used as pharmacodynamic biomarkers [3]. In a study by Rini et al., serum levels of these factors were measured before and during treatment with VEGF-targeted therapy. Patients with baseline levels of sVEGFR-3 were associated with a better prognosis, suggesting that these factors can be used as predictive markers in this type of therapy [11]. Also, VEGF is a downstream marker of HIF activation, making it a marker for VHL loss and HIF deregulation [3].

CIAX is a protein induced by hypoxia, which is widely found in RCC cells. This protein regulates the conversion of carbon dioxide to carbonic acid, leading to $\mathrm{pH}$ homeostasis in tumors during hypoxic conditions and the accumulation of lactic acid [3]. CIAX has proved to be an effective prognostic marker. In a study by Sandlund et al., RCC patients were evaluated using tissue microarray analysis and CAIX staining; patients with more than $91 \%$ of staining were associated with a better prognosis than patients with low amounts of staining $[12,13]$. In another study by Li et al., serum levels of CAIX were analyzed in patients; the level of CAIX was statistically significant in relation to tumor stage, grade, and size [14]. CAIX seems to be a sound marker for prognostic purposes and for VHL/HIF deregulation.

There are further molecules that can be considered as diagnostic and prognostic markers of kidney tumors. These include HIF-1, Survivin, mTOR, CA9, PTEN, tyrosine kinases Akt and S6K, cytokines CCL5 and CXCL9, caveolin-1, and some others. However, most of these proteins are not specific for kidney pathology. For example, Survivin, belonging to the family of apoptosis inhibitors, can be considered as a ubiquitous marker of various cancers, since its expression cannot be detected in differentiated cells of an adult organism and remains detectable only in dividing cells. Quantitative analysis of its expression can also be used as a marker of poor prognosis [15]. Inactivation of PTEN phosphatase is as well typical for the majority of tumor types-glioma, meningioma, melanoma, kidney tumor, hepatoma, cervical, mammary, and prostate carcinoma, so that this marker cannot be considered specific too. Tyrosinkinases Akt and S6K are the components of mTOR signaling pathway, regulating initiation of protein translation in the cell, being responsible for general cell metabolism. Literature data indicate that analysis of pAkt and pS6K expression level in 20 patients undergoing temsirolimus therapy can be used for prediction of the efficacy of mTOR-targeting therapy [16]. Expression of caveolin-1, the component of membrane caveole, is associated with bad prognosis of prostate, lung, mammary gland, kidney, and esophagus tumors [17]. Protooncogene $\mathrm{Bcl}-2$ is a candidate marker of a good prognosis in kidney cancer. In a study of 101 cases of kidney tumors, a correlation with good prognosis of disease was observed [18]. However, there are other studies, indicating that there is no correlation between $\mathrm{Bcl}-2$ and prognosis of the disease $[19,20]$. More specific seems to be the analysis of several markers. For instance, simultaneous increase of caveolin and the components of Akt/ mTOR-mediated signaling is a strong indicator of poor prognosis of kidney cancer [21].

In addition to mutations, epigenetic gene regulation by modifying DNA methylation is a further major mechanism of cancer initiation and progression. There is clear evidence that DNA hypermethylation of CpG islands around the promoter regions silences tumor-suppressor genes [22]. This process in turn closely couples with and depends on histone deacetylation. In fact, abnormal histone acetylation status has been demonstrated to initiate undesirable phenotypic changes and cancer [23]. Hence, histone deacetylases (HDAC) inhibitors are supposed to be useful in cancer prevention, due to their ability to 
"reactivate" the expression of epigenetically silenced genes, including those involved in differentiation, invasion, and metastasis. During the last years, it was shown that HDAC blockade significantly reduces RCC growth and invasion in vitro and in vivo [24-26]. Nevertheless, downregulating HDAC alone seems not to be sufficient to induce long-lasting antitumor effects in clinical trials. It is therefore necessary to further optimize the treatment protocol, either by introducing an additional compound $[27,28]$ or by identifying very specific target structures, manipulation of which strongly stops progressive RCC dissemination.

\section{Immune escape}

It is believed that RCC is partially controlled by the immune system because of the frequency of the disease in immunosuppressed people, the amount of $\mathrm{T}$ cell infiltration to the tumor sites, and the ability of metastasis in these individuals to regress spontaneously. Immune escape in these tumors is caused by alterations in immune cells, the tumor microenvironment, tumor loss of function, and tumor gain of function [29].

Around $40 \%$ of RCC lesions have at least a partial loss of MHC class I antigens, and $6 \%$ show a complete loss [30]. MHC class I antigen processing and presentation is important for the recognition of tumor cells by CD8+ cytotoxic $\mathrm{T}$ lymphocytes (CTLs). Other molecules involved in antigen presentation, such as the transporterassociated with antigen processing (TAP) subunit 1, the chaperone tapasin, and low-molecular weight protein 2 and 7 (LMP2 and LMP7) are also regularly downregulated in RCC cells in comparison to surrounding normal kidney epithelium [29]. It has also been found that the RCC cells lack costimulatory molecules B7-1 and B7-2. The lack of these cells causes deficient $\mathrm{T}$ cell activation leading to $\mathrm{T}$ cell anergy and apoptosis.

RCC cells also escape immunity due to gains in function. HLA-G has been found to be upregulated in RCC. This protein is involved in the immune system by interacting with immunoglobulin-like killer inhibitory receptors (KIRs) that are found on natural killer (NK) cells and T lymphocytes [29]. When HLA-G is bound to these receptors, it protects tumor cells from attack from these cells. Effector cells can also be killed due to an increase in apoptosis-inducing molecules. It was found that when $\mathrm{T}$ cells were cocultivated with RCC cells, more T-cells went into apoptosis than normal. This may be because of an upregulation of tumor necrosis factor (TNF)-related apoptosis inducing ligand (TRAIL), Fas ligand (FasL), or CD70 [31].

Alterations in T cells and other immune cells also lead to a decreased immune response in RCC patients. DCs are poorly recruited to tumor tissues, and T cells are silenced by the secretion of factors involved in the TCR signaling transduction pathways. A downregulation of the TCR zeta chain and p56 (Ick) has been found in the tumor infiltrating lymphocytes of these patients, impairing $\mathrm{T}$ cell activation [29].

The tumor microenvironment plays highly important role in the RCC cells' ability to escape immune responses. The infiltration of lymphocytes, tumor-associated macrophages, and DCs to the tumor sites contributes to the tumor. These cells are able to produce factors such as TNF- $\alpha$ or IL- 6 which promote tumor growth and IFN, IL4 , or IL-13 that prevent proliferation [29, 31].

\section{Tumor-associated macrophages in kidney cancer}

The tumor is comprised of a heterogeneous microenvironment of both malignant and normal stromal cells [32] and contains a large amount of macrophages called tumor-associated macrophages (TAMs). These macrophages have the ability to reduce tumor growth via non-specific cytotoxic mechanisms or specific cell lysis [33, 34]. However, in most of the cases, TAMs are modified in a way to aid in malignant cell progression, invasiveness, and evasion of apoptosis [32]. Chemokines such as monocyte chemotactic protein-1 (MCP-1), macrophage colony stimulating factor (M-CSF), and VEGF are secreted by the tumor cells and attract macrophages to the site of malignancy [35].

A variety of traits possessed by macrophages can then aid in the progression of the tumor. One example is the macrophage's ability to aid in angiogenesis through the release of angiogenic factors, such as cytokines and matrix metalloproteinases. Tumors need angiogenesis in order to grow and for metastasis. The process of angiogenesis includes extracellular matrix degradation, proliferation and migration of capillary endothelial cells and differentiation of these cells into mature capillaries, providing a route for nutrients and oxygen to enter the tumor and for metastatic cells to exit the tumor into circulation [35].

Tumor-associated macrophages have also been shown to aid in tumor growth. These macrophages secrete various factors such as EGF, TGF- $\beta 1$, and basic FGF when cocultured with tumor cells aiding in proliferation and survival. Tumor-associated macrophages have also been shown to be essential for tumor development in macrophage depletion studies [36].

It has been shown that macrophages exposed to apoptotic tumor cells have reduced cytotoxicity and nitric oxide production in response to interferon-gamma and lipopolysaccharide. It has also been shown that macrophages exposed to apoptotic tumor cells increase the growth of tumor cells by $40 \%$ and tumor-associated macrophages promote tumor angiogenesis, invasion, intravasation, and metastasis in animal models [37]. 
In kidney tumors, an association between the amount of TAMs is well established. It was demonstrated that high infiltration of tumor with CD163+ cells correlates with poor clinical prognosis [38]. In the same study, the authors demonstrate that direct coculture of macrophages with RCC cells induces type 2 macrophage phenotype. This is explained by the expression of membrane-type MCSF on the surface of RCC cells. At the same time, it was shown that TAMs isolated out of RCC express not only immunosuppressive IL-10 but also pro-inflammatory chemokine CCL2 [39]. These macrophages also showed enhanced eicosanoid production via activated 15lipolxygenase-2 pathway [39], what is typical for activation of macrophages by TGF $\beta$ [40]. Though unexpected, another specific property of TAMs in RCC is expression of CCR8 associated with higher activity of Stat3-mediated signaling, what is rather typical for inflammatory phenotype. These cells are considered to be capable of stimulating FoxP3 expression in $\mathrm{T}$ cells and have proangiogenic activity [41]. There are more confirmations of proinflammatory phenotype of TAMs in RCC. Petrella and Vincenti reported that increased expression of IL1 $\beta$, a cytokine that may be produced by inflammatory macrophages, contribute to aggressive RCCs. This cytokine leads to expression of matrix metalloproteinases (MMPs) $-1,-3$, and -10 that contribute to tumor cell invasion [42].

There is also a connection between TAM density and VEGF pathway. In the study by Toge et al., it was found that in more progressive RCC, the VEGF level was higher what correlated with higher numbers of TAM and higher microvessel density. Also, VEGF level and TAM were significantly higher in patients with recurrence. The authors identified VEGF and TAM numbers as prognostic factors. Moreover, TAM was the only independent prognostic factor by multivariate analysis [43]. These data are supported by the study, demonstrating that VEGFR1 knockdown leads to reduced macrophage infiltration in the tumor [44].

It is clear that RCC TAMs show mixed phenotype what makes the analysis of the balance between M1 and M2 type activation even more important. In the paper of $\mathrm{Xu}$ et al., it was demonstrated that the number of TAMs has only limited prognostic value while the analysis of CD11c/ CD206 balance provides a good possibility to determine individual prognosis (low CD11c and high CD206 densities are associated with poor prognosis) [45].

Although most of the studies are performed on clear cell RCC, the importance of TAMs was demonstrated also for papillary renal cell carcinoma. Papillary RCC can be subdivided in subtypes I and II based on histological criteria. Type II is associated with poor prognosis. Although density of TAM CD68+ tam was similar in both subtypes, the analysis of more specific markers revealed that nearly all macrophages in type II tumors express CD163, while in type I tumors, there were less than $30 \%$ CD163 positive. Higher number of CD163 cells was associated with higher density of capillaries as defined by CD31 staining. These findings suggest the functional impact of TAMs on the prognosis of papillary RCC type II [46].

\section{Personalized therapy}

Since the loss of VHL expression (50-80 \% cases of clear cell renal cell carcinoma) is the most frequent molecular event observed in kidney tumors, most of the targeted drugs used for the treatment of this pathology are inhibitors of various components of signaling pathways related to VHL inactivation. However, it is important to note that kidney tumors other than renal cell carcinoma, i.e., papillary, chromophobe, collective duct, and other types of renal cell carcinoma are not associated with VHL inactivation. Therefore, search for new molecular markers and signaling pathways which components can become therapeutic targets is necessary for effective treatment of RCC.

The currently available therapeutic agents for advanced RCC include one monoclonal antibody (bevacizumab), four TKIs targeting VEGF receptors (VEGFRs) (sorafenib, sunitinib, pazopanib, and axitinib), two inhibitors of the mammalian target of rapamycin-related complexes (temsirolimus and everolimus), and one recombinant form of an endogenous cytokine (interleukin-2). At present, sunitinib and pazopanib are the most frequently used first-line treatments for advanced RCC. However, $25 \%$ of patients receiving this treatment will not obtain any benefit, demonstrating early progression of the disease. Secondary, resistance is also an important problem, as most patients who initially respond to these agents will eventually progress [47].

To increase RCC therapy efficacy, targeted drugs are combined with antiangiogenic ones. The latter include drugs that block HIF1 $\alpha$-VEGF signaling at several levels, for example, combination of drugs that block VEGF and its receptors. Other type of combined drug includes blocking of several signaling pathways simultaneously, i.e., VEGF, PDGF, EGF signaling pathways and mTOR pathway $[48,49]$.

Several germline studies have investigated the effect of polymorphisms on the efficacy and toxicity of antiangiogenic agents, mainly focusing on genes-encoding enzymes involved in metabolism (CYP3A4, CYP3A5, and their regulator NR1I3), transporters (ABCB1 and $A B C G 2)$, and targets (VEGF, VEGFRs, and FLT3) of these drugs. The first pharmacogenetic study of sunitinib supported the impact of SNPs in NR1I3, ABCB1, and CYP3A5 on progression-free survival [50], and a subsequent study revealed an association between two VEGFR3 missense 
polymorphisms and poor outcome with sunitinib treatment [51].

In a recent study of patients treated with pazopanib, $\mathrm{Xu}$ et al. [52] found an association between progression-free survival and SNPs in IL8 and HIF1A, and the association between UGT1A1 polymorphisms and bilirubin elevation in pazopanib-treated patients has recently been confirmed. Also, higher responses to antiangiogenic drugs were reported in familial VHL-RCC (i.e., RCC with germline $V H L$ mutations) in some but not all studies [53-55].

Despite active development of therapeutic approaches for RCC, it remains unclear how to select the optimal therapy for a particular patient. In contrast to adenocarcinoma of the lung or colon where mutations in EGFR or $K-R A S$ are the markers clearly predicting the sensitivity of tumor cells to EGFR inhibitors, the presence of VHL mutations is not a clear indication for the use of VEGF/ VEGFR inhibitors or other antiangiogenic drugs. It is not yet clear which VHL mutations lead to a complete loss of function $[56,57]$.

Therefore to date, the clinical benefit an individual patient will derive from antiangiogenic therapy is highly variable and largely unpredictable. Between 20 and $30 \%$ of patients with ccRCC derive no benefit from first-line TKI treatment $[47,58]$. In addition, these drugs are toxic and expensive. There is thus great impetus to discover biomarkers in RCC that can identify the subpopulation of patients destined to gain maximal benefit from any given drug.

\section{Expert recommendation}

Molecular markers, identified during last years provide an excellent possibility for the development of a personalized and predictive approach for patient stratification and development of treatment strategy. Classification of these markers according to the recommendations of EPMA provided in the section "Biomarker Discovery, Validation, Standardisation and Practical Application in Medical Practice" of the EPMA White Paper [59] will improve the usage of these markers in clinical practice.

Data is available for genetic and epigenetic changes in the tumor cells and immunologic properties of the tumor. A complex study that will include investigation of all these parameters, i.e., mutations, gene silencing by DNA methylation, and properties of TAMs is needed. Special attention has to be given to tumor-associated macrophages. Research papers indicate that simple measurement of TAMs amount provides insufficient information; therefore, additional research is needed to collect more information about favorable and detrimental macrophage phenotypes. It is important not to limit the analysis to simple discrimination between M1 and M2 macrophage phenotype but to identify the effects of individual cytokines in connection with personal properties of the immune system. This can be achieved using the following markers: CD68, for general macrophage identification; CD163, mannose receptor; Stab1, for general M2 identification; fibronectin, $\beta \mathrm{IG}-\mathrm{H} 3, \mathrm{MMP} 2$, and MMP12, for IL4-induced effects; Id3, OLR1, HAMP, and YKL-39, for TGF $\beta$-induced effects; MCP1, MCP2, and TNF, for M1 identification.

The early diagnosis of RCC, however, remains to be illusive. There are no reliable soluble markers that can be detected in the blood or urine so far. Therefore, more complex strategies have to be developed for early detection or even prediction of RCC in accordance with the principles of preventive and personalized medicine (PPPM) [60]. These may include analysis of mutation and methylation of genes involved in RCC pathogenesis in circulating tumor cells or in plasma DNA.

\section{Competing interests}

The authors declare that they have no competing interests.

\section{Authors' contributions}

AM and OK drafted the manuscript, JK edited the manuscript, and AG conceived the study, coordinated drafting of the manuscript, and finalized the manuscript. All authors read and approved the final manuscript.

\section{Acknowledgements}

This research was supported by RSCF grant, project 14-15-00396 (AG) and by Tomsk State University Competitiveness Improvement Program (JK).

\section{Author details}

${ }^{1}$ Institute of Transfusion Medicine and Immunology, Medical Faculty Mannheim, Ruprecht-Karls University of Heidelberg, Mannheim, Germany. ${ }^{2}$ Blokhin Cancer Research Center, Moscow, Russia. ${ }^{3}$ Red Cross Blood Service Baden-Württemberg-Hessen, Mannheim, Germany. ${ }^{4}$ Laboratory for Translational Cellular and Molecular Biomedicine, Tomsk State University, Tomsk, Russia. ${ }^{5}$ Laboratory of the Tumour Stromal Cells Biology, Institute of Carcinogenesis, Blokhin Cancer Research Center, Kashirskoye Shosse 24, Moscow, Russia.

Received: 19 August 2015 Accepted: 18 September 2015

Published online: 20 October 2015

\section{References}

1. Bukowski RM, Olencki T, Wang Q, Peereboom D, Budd GT, Elson P, et al. Phase II trial of interleukin-2 and interferon-alpha in patients with renal cell carcinoma: clinical results and immunologic correlates of response. J Immunother. 1997;20(4):301-11.

2. Yu MC, Mack TM, Hanisch R, Cicioni C, Henderson BE. Cigarette smoking, obesity, diuretic use, and coffee consumption as risk factors for renal cell carcinoma. J Natl Cancer Inst. 1986;77(2):351-6.

3. Garcia JA, Cowey CL, Godley PA. Renal cell carcinoma. Curr Opin Oncol. 2009:21(3):266-71.

4. Braga VA. Teaching the renal tubular reabsorption of glucose using two classic papers by Shannon et al. Adv Physiol Educ. 2011;35(2):114-6.

5. Rini BI, Campbell SC, Escudier B. Renal cell carcinoma. Lancet. 2009;373(9669):1119-32.

6. Iliopoulos O, Levy AP, Jiang C, Kaelin Jr WG, Goldberg MA. Negative regulation of hypoxia-inducible genes by the von Hippel-Lindau protein. Proc Natl Acad Sci U S A. 1996;93(20):10595-9.

7. Turcotte S, Chan DA, Sutphin PD, Hay MP, Denny WA, Giaccia AJ. A molecule targeting $\mathrm{VHL}$-deficient renal cell carcinoma that induces autophagy. Cancer Cell. 2008;14(1):90-102.

8. Sultmann $H$, von Heydebreck $A$, Huber $W$, Kuner $R$, Buness A, Vogt $M$ et al. Gene expression in kidney cancer is associated with cytogenetic abnormalities, metastasis formation, and patient survival. Clin Cancer Res. 2005;11(2 Pt 1):646-55. 
9. Yao M, Yoshida M, Kishida T, Nakaigawa N, Baba M, Kobayashi K, et al. VHL tumor suppressor gene alterations associated with good prognosis in sporadic clear-cell renal carcinoma. J Natl Cancer Inst. 2002;94(20):1569-75.

10. Choueiri TK, Vaziri SA, Jaeger E, Elson P, Wood L, Bhalla IP, et al. von HippelLindau gene status and response to vascular endothelial growth factor targeted therapy for metastatic clear cell renal cell carcinoma. J Urol. 2008;180(3):860-5. discussion 5-6.

11. Rini BI, Rathmell WK, Godley P. Renal cell carcinoma. Curr Opin Oncol. 2008;20(3):300-6

12. Sandlund J, Oosterwijk E, Grankvist K, Oosterwijk-Wakka J, Ljungberg B, Rasmuson T. Prognostic impact of carbonic anhydrase IX expression in human renal cell carcinoma. BJU Int. 2007;100(3):556-60.

13. Rathmell WK, Godley PA. Recent updates in renal cell carcinoma. Curr Opin Oncol. 2010;22(3):250-6.

14. Li G, Feng G, Gentil-Perret A, Genin C, Tostain J. Serum carbonic anhydrase 9 level is associated with postoperative recurrence of conventional renal cell cancer. J Urol. 2008;180(2):510-3. discussion 3-4.

15. Zamparese R, Pannone G, Santoro A, Lo Muzio L, Corsi F, Pedicillo MC, et al. Survivin expression in renal cell carcinoma. Cancer Invest. 2008;26(9):929-35.

16. Cho D, Signoretti S, Dabora S, Regan M, Seeley A, Mariotti M, et al. Potential histologic and molecular predictors of response to temsirolimus in patients with advanced renal cell carcinoma. Clin Genitourin Cancer. 2007:5(6):379-85.

17. Hehlgans S, Cordes N. Caveolin-1: an essential modulator of cancer cell radio-and chemoresistance. Am J Cancer Res. 2011;1(4):521-30.

18. Itoi T, Yamana K, Bilim V, Takahashi K, Tomita F. Impact of frequent Bcl-2 expression on better prognosis in renal cell carcinoma patients. Br J Cancer. 2004;90(1):200-5.

19. Vasavada SP, Novick AC, Williams BR. P53, bcl-2, and Bax expression in renal cell carcinoma. Urology. 1998;51(6):1057-61.

20. Uchida T, Gao JP, Wang C, Jiang SX, Muramoto M, Satoh T, et al. Clinical significance of $\mathrm{p} 53, \mathrm{mdm} 2$, and bcl-2 proteins in renal cell carcinoma. Urology. 2002;59(4):615-20.

21. Campbell L, Jasani B, Edwards K, Gumbleton M, Griffiths DF. Combined expression of caveolin-1 and an activated AKT/mTOR pathway predicts reduced disease-free survival in clinically confined renal cell carcinoma. Br J Cancer. 2008;98(5):931-40.

22. Arai $\mathrm{E}$, Kanai $\mathrm{Y}$. Genetic and epigenetic alterations during renal carcinogenesis. Int J Clin Exp Pathol. 2010;4(1):58-73.

23. Mottet D, Castronovo V. Histone deacetylases: target enzymes for cancer therapy. Clin Exp Metastasis. 2008;25(2):183-9.

24. Jones J, Juengel E, Mickuckyte A, Hudak L, Wedel S, Jonas D, et al. The histone deacetylase inhibitor valproic acid alters growth properties of renal cell carcinoma in vitro and in vivo. J Cell Mol Med. 2009;13(8B):2376-85

25. Jones J, Juengel E, Mickuckyte A, Hudak L, Wedel S, Jonas D, et al. Valproic acid blocks adhesion of renal cell carcinoma cells to endothelium and extracellular matrix. J Cell Mol Med. 2009;13(8B):2342-52.

26. Juengel E, Bhasin M, Libermann T, Barth S, Michaelis M, Cinatl Jr J, et al. Alterations of the gene expression profile in renal cell carcinoma after treatment with the histone deacetylase-inhibitor valproic acid and interferon-alpha. World J Urol. 2011;29(6):779-86.

27. Juengel E, Engler J, Mickuckyte A, Jones J, Hudak L, Jonas D, et al. Effects of combined valproic acid and the epidermal growth factor/vascular endothelial growth factor receptor tyrosine kinase inhibitor AEE788 on renal cell carcinoma cell lines in vitro. BJU Int. 2010;105(4):549-57.

28. Juengel E, Engler J, Natsheh I, Jones J, Mickuckyte A, Hudak L, et al. Combining the receptor tyrosine kinase inhibitor AEE788 and the mammalian target of rapamycin (mTOR) inhibitor RAD001 strongly inhibits adhesion and growth of renal cell carcinoma cells. BMC Cancer. 2009;9:161

29. Seliger B, Schlaf G. Structure, expression and function of HLA-G in renal cell carcinoma. Semin Cancer Biol. 2007;17(6):444-50.

30. Romero JM, Aptsiauri N, Vazquez F, Cozar JM, Canton J, Cabrera T, et al. Analysis of the expression of HLA class I, proinflammatory cytokines and chemokines in primary tumors from patients with localized and metastatic renal cell carcinoma. Tissue Antigens. 2006;68(4):303-10.

31. Seliger B, Dressler SP, Lichtenfels R, Kellner R. Candidate biomarkers in renal cell carcinoma. Proteomics. 2007;7(24):4601-12.

32. Van Ginderachter JA, Movahedi K, Hassanzadeh Ghassabeh G, Meerschaut S, Beschin A, Raes $G$, et al. Classical and alternative activation of mononuclear phagocytes: picking the best of both worlds for tumor promotion. Immunobiology. 2006;211(6-8):487-501.
33. Blachere NE, Li Z, Chandawarkar RY, Suto R, Jaikaria NS, Basu S, et al. Heat shock protein-peptide complexes, reconstituted in vitro, elicit peptidespecific cytotoxic T lymphocyte response and tumor immunity. J Exp Med. 1997;186(8):1315-22.

34. Grabbe S, Bruvers S, Beissert S, Granstein RD. Interferon-gamma inhibits tumor antigen presentation by epidermal antigen-presenting cells. J Leukoc Biol. 1994;55(6):695-701.

35. Bingle $L$, Brown NJ, Lewis CE. The role of tumour-associated macrophages in tumour progression: implications for new anticancer therapies. J Pathol. 2002;196(3):254-65.

36. Wu D, Molofsky AB, Liang HE, Ricardo-Gonzalez RR, Jouihan HA, Bando JK, et al. Eosinophils sustain adipose alternatively activated macrophages associated with glucose homeostasis. Science. 2011;332(6026):243-7.

37. Reiter I, Krammer B, Schwamberger G. Cutting edge: differential effect of apoptotic versus necrotic tumor cells on macrophage antitumor activities. J Immunol. 1999;163(4):1730-2.

38. Komohara $Y$, Hasita H, Ohnishi K, Fujiwara Y, Suzu S, Eto M, et al. Macrophage infiltration and its prognostic relevance in clear cell renal cell carcinoma. Cancer Sci. 2011;102(7):1424-31.

39. Daurkin I, Eruslanov E, Stoffs T, Perrin GQ, Algood C, Gilbert SM, et al. Tumor-associated macrophages mediate immunosuppression in the renal cancer microenvironment by activating the 15-lipoxygenase-2 pathway. Cancer Res. 2011;71(20):6400-9.

40. Gratchev A, Kzhyshkowska J, Kannookadan S, Ochsenreiter M, Popova A, Yu X, et al. Activation of a TGF-beta-specific multistep gene expression program in mature macrophages requires glucocorticoid-mediated surface expression of TGF-beta receptor II. J Immunol. 2008;180(10):6553-65.

41. Eruslanov E, Stoffs T, Kim WJ, Daurkin I, Gilbert SM, Su LM, et al. Expansion of CCR8(+) inflammatory myeloid cells in cancer patients with urothelial and renal carcinomas. Clin Cancer Res. 2013;19(7):1670-80.

42. Petrella BL, Vincenti MP. Interleukin-1beta mediates metalloproteinasedependent renal cell carcinoma tumor cell invasion through the activation of CCAAT enhancer binding protein beta. Cancer Med. 2012;1(1):17-27.

43. Toge $H$, Inagaki $T$, Kojimoto $Y$, Shinka $T$, Hara I. Angiogenesis in renal cell carcinoma: the role of tumor-associated macrophages. Int J Urol. 2009;16(10):801-7

44. Li C, Liu B, Dai Z, Tao Y. Knockdown of VEGF receptor-1 (VEGFR-1) impairs macrophage infiltration, angiogenesis and growth of clear cell renal cell carcinoma (CRCC). Cancer Biol Ther. 2011;12(10):872-80.

45. Xu L, Zhu Y, Chen L, An H, Zhang W, Wang G, et al. Prognostic value of diametrically polarized tumor-associated macrophages in renal cell carcinoma. Ann Surg Oncol. 2014;21(9):3142-50.

46. Behnes CL, Bremmer F, Hemmerlein B, Strauss A, Strobel P, Radzun HJ. Tumor-associated macrophages are involved in tumor progression in papillary renal cell carcinoma. Virchows Arch. 2014;464(2):191-6.

47. Motzer RJ, Hutson TE, Tomczak P, Michaelson MD, Bukowski RM, Rixe O, et al. Sunitinib versus interferon alfa in metastatic renal-cell carcinoma. N Eng J Med. 2007;356(2):115-24.

48. Sosman J, Puzanov I. Combination targeted therapy in advanced renal cell carcinoma. Cancer. 2009;115(10 Suppl):2368-75.

49. Bellmunt J, Pons F, Foreshew A, Fay AP, Powles T, Porta C, et al. Sequential targeted therapy after pazopanib therapy in patients with metastatic renal cell cancer: efficacy and toxicity. Clin Genitourin Cancer. 2014;12(4):262-9.

50. van der Veldt AA, Eechoute K, Gelderblom H, Gietema J, Guchelaar HJ, van Erp NP, et al. Genetic polymorphisms associated with a prolonged progression-free survival in patients with metastatic renal cell cancer treated with sunitinib. Clin Cancer Res. 2011;17(3):620-9.

51. Garcia-Donas J, Esteban E, Leandro-Garcia L, Castellano DE, del Alba AG, Climent MA, et al. Single nucleotide polymorphism associations with response and toxic effects in patients with advanced renal-cell carcinoma treated with first-line sunitinib: a multicentre, observational, prospective study. Lancet Oncol. 2011;12(12):1143-50.

52. Xu CF, Bing NX, Ball HA, Rajagopalan D, Sternberg CN, Hutson TE, et al. Pazopanib efficacy in renal cell carcinoma: evidence for predictive genetic markers in angiogenesis-related and exposure-related genes. J Clin Oncol. 2011:29(18):2557-64.

53. Kim HC, Lee JS, Kim SH, So HS, Woo CY, Lee JL. Sunitinib treatment for metastatic renal cell carcinoma in patients with von Hippel-Lindau disease. Cancer Res Treat. 2013;45(4):349-53.

54. Tsimafeyeu I, Demidov L. Treatment options for renal cell carcinoma in patients with von Hippel-Lindau disease. J Cancer Res Ther. 2010;6(4):575-7. 
55. Jonasch E, McCutcheon IE, Waquespack SG, Wen S, Davis DW, Smith LA, et al. Pilot trial of sunitinib therapy in patients with von Hippel-Lindau disease. Ann Oncol. 2011;22(12):2661-6.

56. Kondo K, Yao M, Yoshida M, Kishida T, Shuin T, Miura T, et al.

Comprehensive mutational analysis of the VHL gene in sporadic renal cell carcinoma: relationship to clinicopathological parameters. Genes Chromosomes Cancer. 2002;34(1):58-68.

57. Kawakami T, Okamoto K, Ogawa O, Okada Y. Multipoint methylation and expression analysis of tumor suppressor genes in human renal cancer cells. Urology. 2003;61(1):226-30.

58. Sternberg CN, Davis ID, Mardiak J, Szczylik C, Lee E, Wagstaff J, et al. Pazopanib in locally advanced or metastatic renal cell carcinoma: results of a randomized phase III trial. J Clin Oncol. 2010;28(6):1061-8.

59. Golubnitschaja O, Costigliola V, EPMA. General report \& recommendations in predictive, preventive and personalised medicine 2012: white paper of the European Association for Predictive, Preventive and Personalised Medicine. EPMA J. 2012:3(1):14.

60. Grech G, Zhan X, Yoo BC, Bubnov R, Hagan S, Danesi R, et al. EPMA position paper in cancer: current overview and future perspectives. EPMA J. 2015;6(1):9.

\section{Submit your next manuscript to BioMed Central and take full advantage of:}

- Convenient online submission

- Thorough peer review

- No space constraints or color figure charges

- Immediate publication on acceptance

- Inclusion in PubMed, CAS, Scopus and Google Scholar

- Research which is freely available for redistribution 